ARTICLE

\title{
Extinct type of human parvovirus B19 persists in tonsillar B cells
}

Lari Pyöriä ${ }^{1, \star}$, Mari Toppinen ${ }^{1, \star}$, Elina Mäntylä2 ${ }^{2}$, Lea Hedman ${ }^{1,3}$, Leena-Maija Aaltonen ${ }^{4}$, Maija Vihinen-Ranta ${ }^{2}$, Taru Ilmarinen 4 , Maria Söderlund-Venermo ${ }^{1}$, Klaus Hedman ${ }^{1,3} \&$ Maria F. Perdomo ${ }^{1}$

Parvovirus B19 (B19V) DNA persists lifelong in human tissues, but the cell type harbouring it remains unclear. We here explore B19V DNA distribution in B, T and monocyte cell lineages of recently excised tonsillar tissues from 77 individuals with an age range of 2-69 years. We show that B19V DNA is most frequent and abundant among B cells, and within them we find a B19V genotype that vanished from circulation $>40$ years ago. Since re-infection or re-activation are unlikely with this virus type, this finding supports the maintenance of pathogen-specific humoral immune responses as a consequence of B-cell long-term survival rather than continuous replenishment of the memory pool. Moreover, we demonstrate the mechanism of B19V internalization to be antibody dependent in two B-cell lines as well as in ex vivo isolated tonsillar B cells. This study provides direct evidence for a cell type accountable for B19V DNA tissue persistence.

\footnotetext{
${ }^{1}$ Department of Virology, University of Helsinki, Helsinki 00290, Finland. ${ }^{2}$ Department of Biological and Environmental Science, and Nanoscience Center, University of Jyväskylä, Jyväskylä 40500, Finland. ${ }^{3}$ Helsinki University Hospital, Helsinki 00290, Finland. ${ }^{4}$ Department of Otorhinolaryngology-Head and Neck Surgery, Helsinki University Hospital, Helsinki 00290, Finland. * These authors contributed equally to this work. Correspondence and requests for materials should be addressed to M.F.P. (email: maria.perdomo@helsinki.fi).
} 
P arvovirus B19 (B19V) infection affects most often children, with exposure rates generally over $50 \%$ by adulthood ${ }^{1}$. The virus circulates worldwide, with current infections mainly due to genotype 1 (ref. 2). Of the other two variants that are known, genotype 2 disappeared from circulation around 1970 (refs 3,4) and genotype 3 has been described to circulate endemically in some regions such as Ghana, Brasil and India ${ }^{5-8}$.

After primary infection, B19V DNA persists lifelong in several human tissues such as tonsils, testicles, kidneys, muscle, salivary glands, thyroid, skin, liver, heart, brain, bone marrow and bone $e^{3,4,9-11}$. However, nothing is known on the specific cell type(s) that harbours it throughout time.

$\mathrm{B} 19 \mathrm{~V}$ replicates in erythroid progenitor cells of the bone marrow with primary infection occurring via the globoside receptor and the $\alpha 5 \beta 1$ integrin and Ku80 co-receptors ${ }^{12-14}$ but uptake has also been shown to occur through antibodydependent enhancement (ADE) in monocytes ${ }^{15}$ and endothelial cells $^{16}$. The short lifetime of these cells, however, does argue against them being the host of this virus' DNA for years after primary infection. Instead, an appealing alternative may be granted by the memory cells that reside in lymphoid organs since their lifespan has been estimated to exceed decades based on the length of immune protection after infection or vaccination $^{17}$.

Hence, in the present study, we evaluate the distribution of B19V DNA in lymphoid cells of recently excised tonsillar tissues. Moreover, we analyse the virus type present, having previously shown ${ }^{11}$ that the $\mathrm{B} 19 \mathrm{~V}$ genotype 2 is a reliable indicator of the age of a tissue. We found the B19V DNA to be primarily distributed in B cells and most importantly, we detected in four adults the extinct genotype 2, thus providing further evidence of this cell type as long-term reservoir of B19V DNA. This finding also enacts as a suitable marker of the longevity of these cells. Moreover, we show ADE to be a mechanism for $\mathrm{B} 19 \mathrm{~V}$ uptake into B cells in vitro.

\section{Results}

B19V DNA persistence in tonsils. B19V DNA has been previously detected in human tonsils ${ }^{3}$ but the specific cell types where it persists lifelong are still unknown. We analysed the B19V-DNA distribution in enriched lymphoid cell suspensions of recently excised tonsillar tissues from 77 individuals who underwent tonsillectomy at the Department of Otorhinolaryngology-Head and Neck Surgery of Helsinki University Hospital. Previous findings by Medina et al. ${ }^{18}$ show that mechanical homogenization alone is not sufficient to release long-lived cells that are associated to connective tissue-rich areas of the tonsil, such as resident plasma cells. Hence a two-step process of tissue homogenization involving mechanical disaggregation followed by collagenase digestion of the residual tonsillar tissue was developed. Each of the resulting cell suspensions was analysed independently for the presence of B19V DNA by an in-house Pan-B19V qPCR amplifying the NS1 region, and the viral copy numbers were normalized to cell counts by quantification of the single copy RNase $P$ gene.

B19V DNA was detected in $26 \%(20 / 77)$ of the total cell populations obtained by mechanical homogenization alone as opposed to $43 \%(33 / 77)$ in those cells released by subsequent collagenase digestion. Moreover, in the latter, the median B19V-DNA copy numbers were 18 -fold higher $(P<0.001$, Mann-Whitney $U$ asymptotic sig. (two-sided test; Fig. 1a)).

The $\mathrm{B}, \mathrm{T}$ and monocyte/macrophage $(\mathrm{M})$ cells were enriched from each tonsillar preparation by positive selection with magnetic beads. The cell fraction purities were: B $96.8 \pm 0.9 \%$, T $95.4 \pm 1.2 \%$, M $93.9 \pm 1.9 \%$ (mean \pm s.d. of 6 replicates).
B19V DNA was preferentially distributed in the B cells of the collagenase-treated preparations (33/33 individuals) which contained also the highest viral loads: median 6.91E1 copies/1E6 cells (95\% confidence interval (CI): 2.26E1-9.53E1 B19V-DNA copies /1E6 cells) as compared to $1.7 \mathrm{E}-1$ copies/1E6 cells (95\% CI: 0.00-3.08) in the fraction resulting from homogenization alone (Fig. 1c). The difference was statistically significant $(P<0.001$, Mann-Whitney $U$ asymptotic sig. (two-sided test)). The B19V-DNA positivity of the B-cell fractions from collagenase-treated tissues was confirmed with a second B19V qPCR amplifying a distinct region (VP1 gene) of the viral genome. There was a strict correlation between both qPCRs, with similar copy numbers (Supplementary Fig. 1).

The Pan-B19V qPCR products of the $\mathrm{B}$ cells released with collagenase were sequenced to determine the persisting B19V genotype. Strikingly, among the six B19V genopositive adults older than 45 years of age (45 to 69; mean 55), four had in their B cells the extinct genotype 2 (median 1.01E2 copies /1E6 cells). All other individuals $(n=29)$ had genotype 1 (Supplementary Table 1).

Interestingly, two children of 6 and 8 years had at least one log higher B19V DNA loads than all the other individuals (3.66E3 and 9.77E3 copies/1E6 cells, respectively). In these two subjects, the highest amounts of B19V DNA were detected in the $M$ population of the homogenized cell suspension (2.50E4 and $5.38 \mathrm{E} 4$ copies /1E6 cells) albeit B19V-DNA levels were high in other cell fractions as well (Supplementary Fig. 2). These two children were IgM negative and non-viremic.

Serum samples available from 74/77 individuals were tested for B19V-IgG and -IgM antibodies. Altogether, 42\% (31/74) were B19V-IgG positive and none were B19V-IgM positive. The B19V-DNA prevalence in tissue and the IgG serostatus of the individuals showed absolute correlation (Supplementary Fig. 3).

Epstein-Barr virus DNA persistence in tonsils. The prevalence of B19V DNA was compared to that of EBV because of the latter's known persistence in B cells ${ }^{19}$.

The EBV-DNA prevalence was $73 \%(24 / 33)$ and $70 \%$ in the B19V DNA-positive and -negative tonsils, respectively, thus lacking significance ( $P=0.827$, Pearson's $\chi^{2}$-test).

EBV DNA was found preferentially distributed in the B cells, with a median of $2.10 \mathrm{E} 2$ and $1.99 \mathrm{E} 2$ copies/1E6 cells among the total cell suspensions obtained with or without collagenase treatment, respectively, in the B19V DNA-positive tonsillar preparations (Fig. 1b,d).

B19V- and EBV-DNA persistence in circulating lymphoid cells. The prevalences of $\mathrm{B} 19 \mathrm{~V}$ and EBV DNA among circulating $B$ cells were evaluated in peripheral blood mononuclear cells (PBMCs) as well as in $\mathrm{CD}^{+} 9^{+}$cells isolated from seven $\mathrm{B} 19 \mathrm{~V}$-seropositive and one $\mathrm{B} 19 \mathrm{~V}$-seronegative, asymptomatic staff members. Both the PBMCs and B cells from all the subjects were PCR-negative for B19V DNA, but positive for EBV DNA in $3 / 8$ individuals (mean $7.60 \mathrm{E} 1$ and $4.81 \mathrm{E} 2$ copies / $1 \mathrm{E} 6$ cells in PBMC and B cells, respectively; Supplementary Table 2).

Compared to tonsillar B cells, the circulating $\mathrm{CD} 19^{+}$cells were globoside negative by flow cytometry (Supplementary Fig. 4d).

Characterization of tonsillar $\mathbf{B}$ cells. The distribution of B19V DNA among naive and memory B cells was studied from collagenase-treated tonsillar cell suspensions of 12 individuals with the highest copy numbers (median 1.06E2 copies/1E6 cells). The cells were stained with anti-CD19 antibodies, to gate the B-cell population, together with CD27 (mature) and IgD (naive) antibodies. Four subpopulations were sorted: $\mathrm{CD} 27^{+}$ 
a

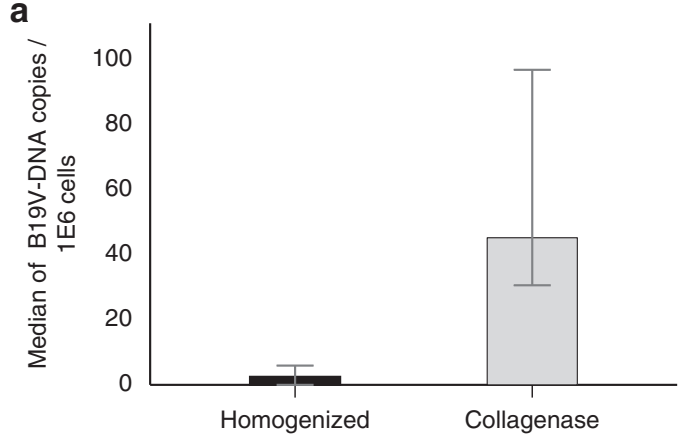

C

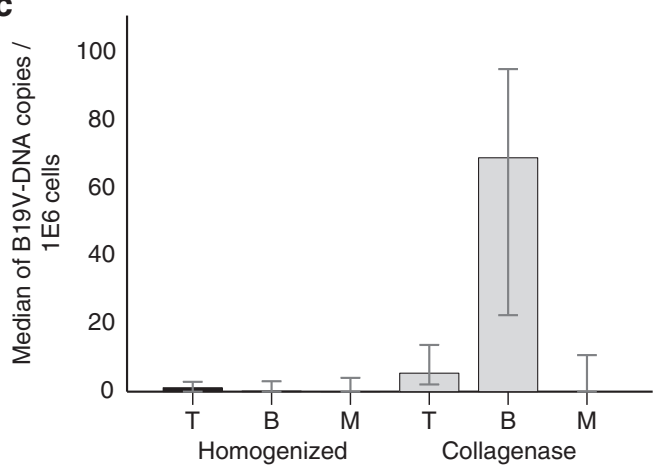

b

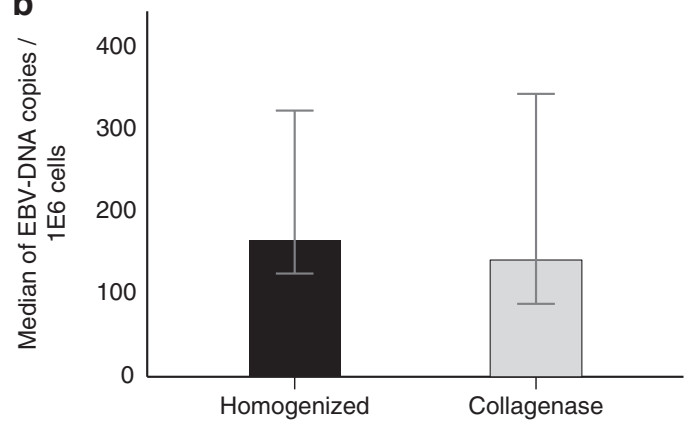

d

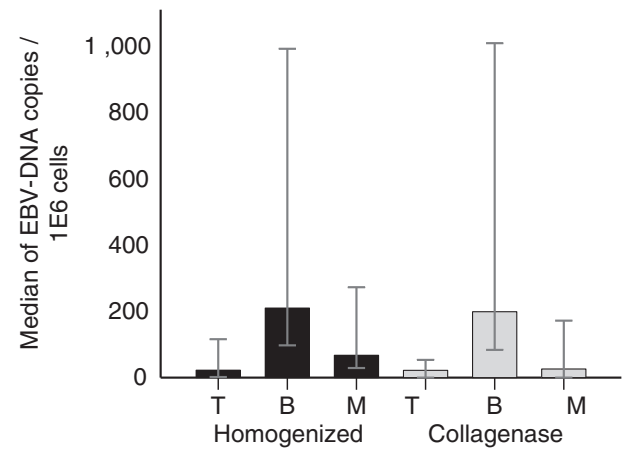

Figure 1 | Viral DNA copies in tonsillar tissue. B19V- and EBV-DNA copies were measured by qPCR and normalized to cell numbers with the human single-copy gene RNase $P$. Top panels: median copy numbers of B19V DNA (a) and EBV DNA (b) in tonsillar cell suspensions resulting from mechanical homogenization $(n=33)$ (black) or after collagenase treatment $(n=33)$ (light grey). The viral copy numbers between the two preparations were significantly different for B19V $(P<0.001)$, while not significant for EBV $(P>0.05)$. Bottom panels: median copy numbers of B19V DNA $(n=33)$ (c) and EBV DNA $(n=24)(\mathbf{d})$ in T-, B- and monocytic cells following homogenization (black) or collagenase digestion (light grey). The viral loads in B cells were statistically significant between preparations for B19V $(P<0.001)$ while not significant for EBV $(P>0.05)$. Error bars indicate $95 \%$ confidence intervals and were determined by normal approximation. Statistical significance was calculated with Mann-Whitney $U$ asymptotic sig. (two-sided test).

$\mathrm{IgD}^{-}, \mathrm{CD}_{2} 7^{+} \mathrm{IgD}^{\text {low }}, \mathrm{CD}_{2} 7^{+} \mathrm{IgD}^{\text {high }}$, and $\mathrm{CD}_{27}^{-} \mathrm{IgD}^{+}$ (Supplementary Fig. 5a). Both the viral loads and cell counts in the fractions were low; the only exception being two children (previously mentioned with the highest copies in the cohort) whose viral DNA copies were one log higher in the $\mathrm{CD} 27^{+}$ IgD ${ }^{\text {high }}$ than in other sub-populations (Supplementary Fig. 5b,c).

Mechanism of B19V entry into B cells. ADE facilitates B19V entry into monocytes ${ }^{15}$ and endothelial cells ${ }^{16}$, although in the latter the process is mediated by proteins of the complement system.

ADE was tested as a possible entry route in two B-cell lines, Raji and GM12878, and on primary tonsillar B cells. These cells were confirmed to express by flow cytometry the Fc-receptor CD32 and the B19V receptor globoside (Supplementary Fig. $4 \mathrm{a}-\mathrm{c}$ ).

The cells were cultured in the presence of either B19V IgG-positive or -negative human sera, as well as of total purified IgG preparations. Virus internalization was followed up both by flow cytometry and confocal microscopy using fluorescent B19 virus-like particles (VLP, non-infectious), consisting of the major capsid protein VP2 (VP2-VLP-AF647). The cells were trypsinized before analysis to trim un-specific signals by non-internalized particles.

In GM12878 and Raji cells, the VLP uptake was detected only in the presence of $\mathrm{B} 19 \mathrm{~V}-\mathrm{IgG}^{+}$sera at dilutions down to $1: 50$ or $1: 100$, respectively, or of corresponding total purified IgGs at $\geq 0.5 \mathrm{mg} \mathrm{ml}^{-1}$ (Fig. 2a-d). Raji cells showed higher and faster uptake efficiency than GM12878 cells.

A reduction in the fluorescence signal of $45.7 \%$ in Raji $(P<0.05$, Student's $t$-test $)$ and of $42.1 \%$ in GM12878 $(P=0.05$,
Student's $t$-test) cells was observed upon blocking of the CD32 molecule with specific antibodies, thus confirming internalization via $\mathrm{Fc}$ receptor (Fig. 2e,f). The VP2-VLP-AF647 uptake was also analysed in the presence of $\mathrm{B} 19 \mathrm{~V}-\mathrm{IgG}^{+}$serum before or after heat-inactivation to evaluate the role of complement. However, no significant difference was observed (Fig. 2c,d).

$\mathrm{ADE}$ was also tested in primary tonsillar B cells from two B19V-seronegative individuals, with capsid uptake detected exclusively in the presence of specific B19V IgGs at concentrations down to $0.5 \mathrm{mg} \mathrm{ml}^{-1}(P<0.01$, Student's $t$-test; Fig. 3a,b). Similar results were obtained with the monocytic cell line U937, in which B19V-ADE was first described, although via the CD64 receptor, thus confirming the suitability of our method to test this mechanism (Fig. 3c).

B19V-capsid internalization was substantiated in both B-cell lines by confocal microscopy (Fig. 4a,d). The VP2-VLP-AF647 capsids localized within early endosome antigen 1 (EEA1)positive endosomes at the cell periphery (Fig. $4 \mathrm{~b}, \mathrm{e}$ ). Line profile analysis confirmed that the VLP and EEA1 localizations were similar in both cell lines (Fig. 4c,f). However, the amount of VLPs in endosomes was higher in the Raji cells than in GM12878 cells. Altogether, our findings suggest that B19-VLPs were able to enter the cells via an endocytic mechanism.

$\mathrm{ADE}$ was evaluated moreover using native ex vivo B19 viruses from a high-titre viremic plasma at 10 particles per cell in the presence of $1 \mathrm{mg} \mathrm{ml}^{-1} \mathrm{~B} 19 \mathrm{~V}$-positive or -negative total purified IgGs (Fig. 5a,b). In the presence of virus-specific antibodies a significant increase in viral DNA copy numbers was observed $(P \leq 0.01$, Student's $t$-test). Moreover, a reduction in virus internalization of $99.6 \%$ in Raji and of $85.6 \%$ in GM12878 cells was measured upon CD32 receptor blockage $(P<0.01$, Student's $t$-test, Fig. $5 c, d)$. 
a

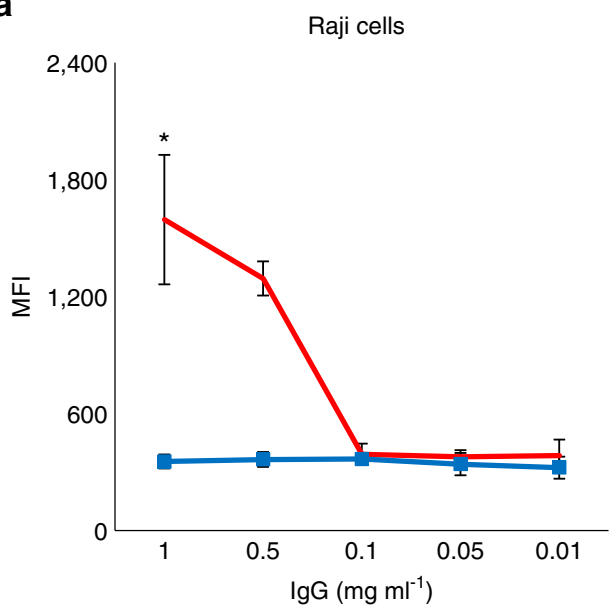

C

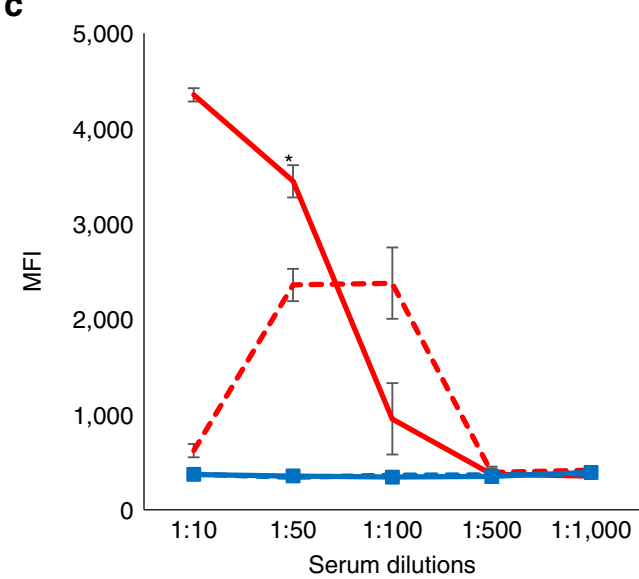

e

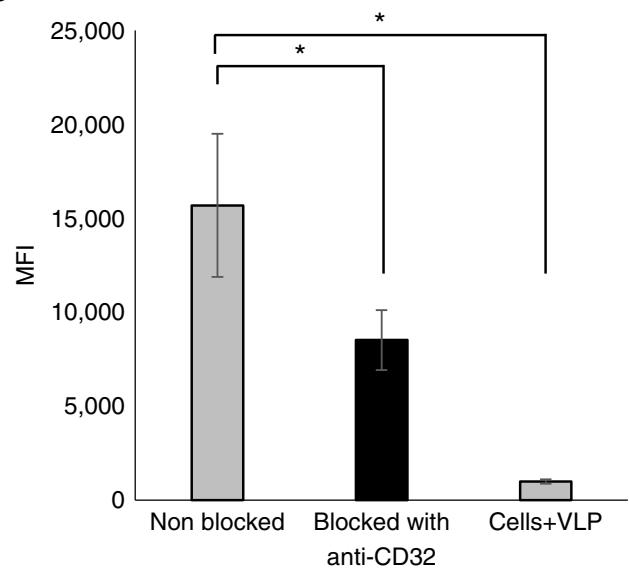

b

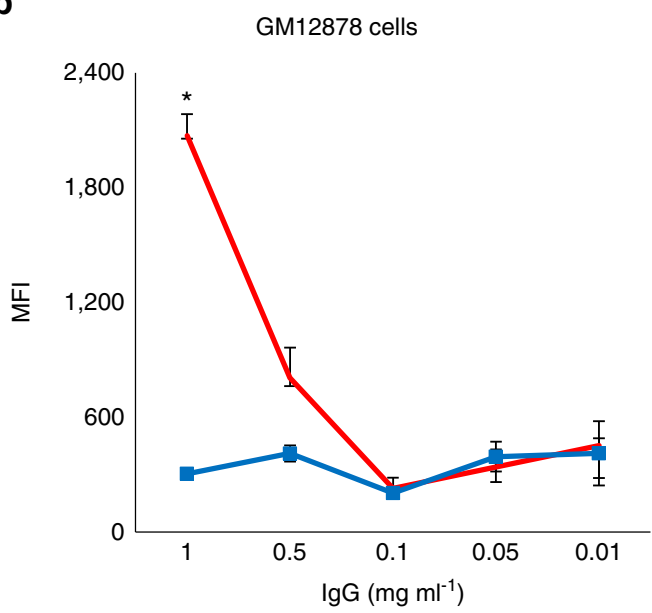

d

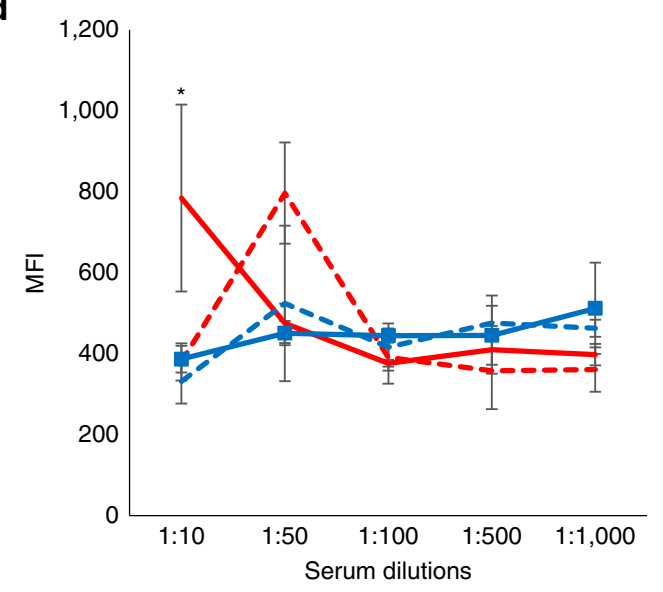

f

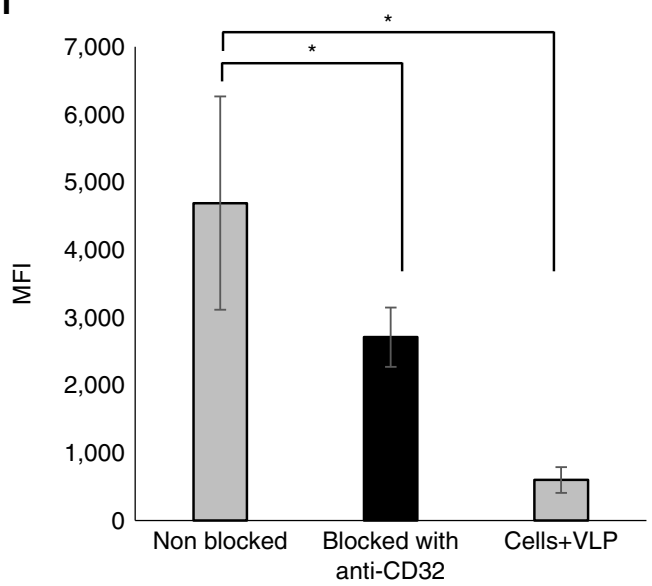

Figure 2 | Antibody-dependent uptake of VP2-VLPs in B cell lines. The mean fluorescence intensity (MFI) of VP2-VLP-AF647 was measured by flow cytometry in Raji (left column) and GM12878 cells (right column) after incubation with total purified B19V-positive (red line) or -negative lgGs (blue line, squares) (a,b). Pooled sera from B19V-lgG ${ }^{+}$individuals either heat inactivated (red solid line) or non-heat inactivated (red dotted line) or pooled sera from B19V-lgG individuals either heat inactivated (blue solid line, squares) or non-heat inactivated (blue dotted line) were used (c, d). ${ }^{*}$ Represents statistical significance $(P<0.001)$ between positive and respective negative controls at a given concentration. The effect on viral uptake was evaluated before (grey bars) and after (black bars) blocking with an anti-CD32 antibody (e,f). The difference was statistically significant (Raji $P<0.001, \mathrm{GM} 12878 P=0.05)$. Error bars represent s.d. (of four replicates). Statistical significance was calculated using Student's $t$-test.

\section{Discussion}

We searched for cell type(s) accounting for lifelong B19V DNA tissue persistence. Hypothesizing that long-lived lymphoid cells may be suitable hosts, we studied the distribution of B19V DNA in tonsillar $\mathrm{B}, \mathrm{T}$ and monocyte cell lineages.
To release long-lived cells that are associated to connective tissue-rich areas of the tonsil ${ }^{18}$, we employed a two-step preparation process of cell suspensions involving mechanical homogenization followed by collagenase digestion. We found that the B19V-DNA loads were significantly higher in the CD19+ 


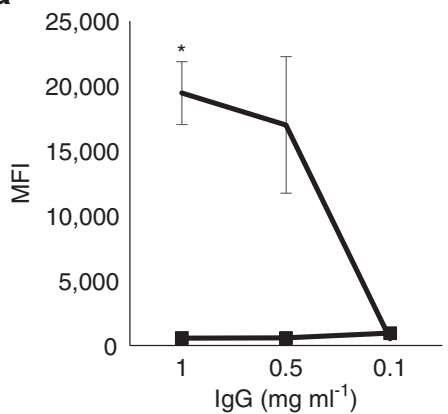

b

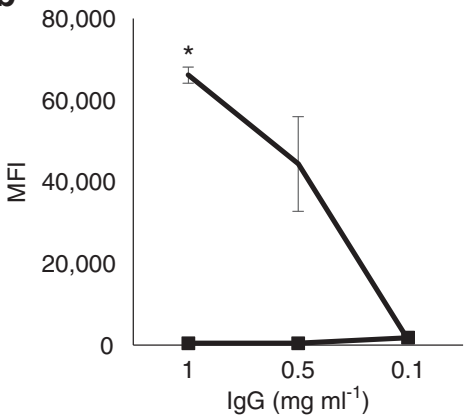

C

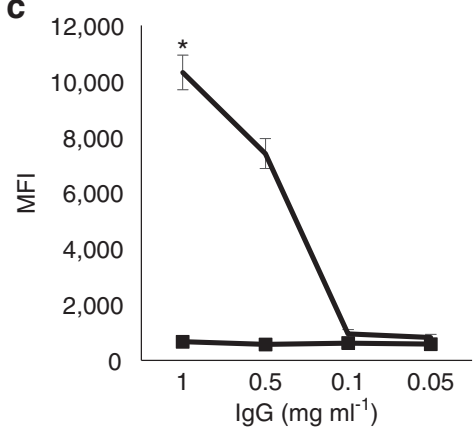

Figure 3 | Antibody-dependent enhancement of VP2-VLPs in primary B cells and in a monocytic cell line. The mean fluorescence intensity (MFI) of VP2-VLP-AF647 was measured by flow cytometry in tonsillar B cells from two seronegative individuals (a,b) as well as in U937 cells (c) after incubation with B19V-lgG ${ }^{+}$(black solid line) or B19V-lgG' (black solid line, squares) total purified preparations. Error bars represent standard deviation (of four replicates). ${ }^{*}$ Represents statistical significance $(P<0.001)$ between positive and negative lgGs at a given concentration as calculated by Student's $t$-test.
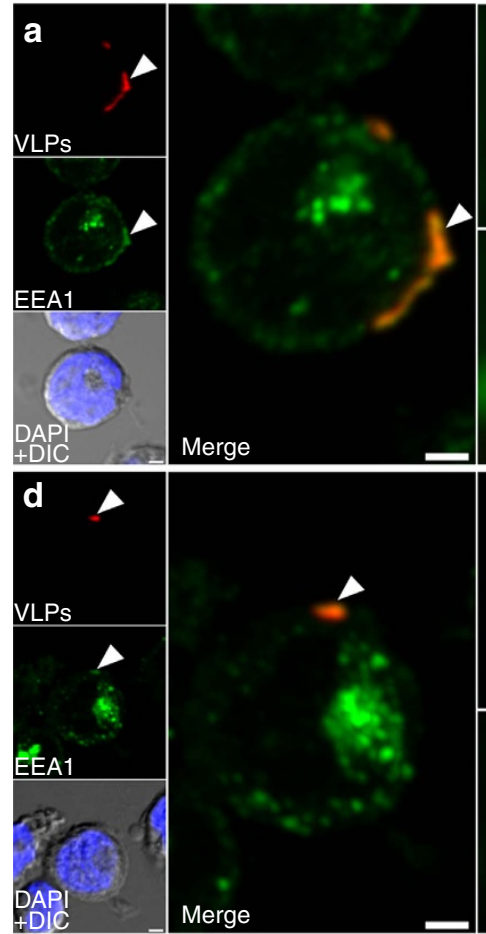

b

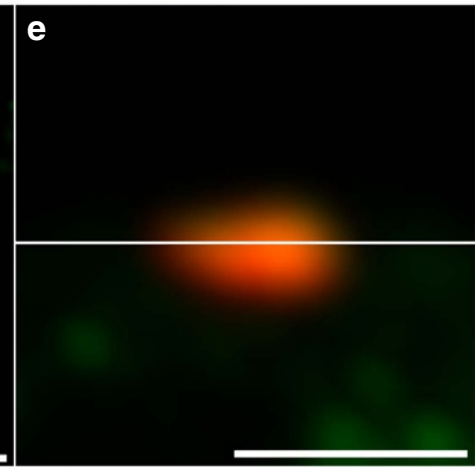

c
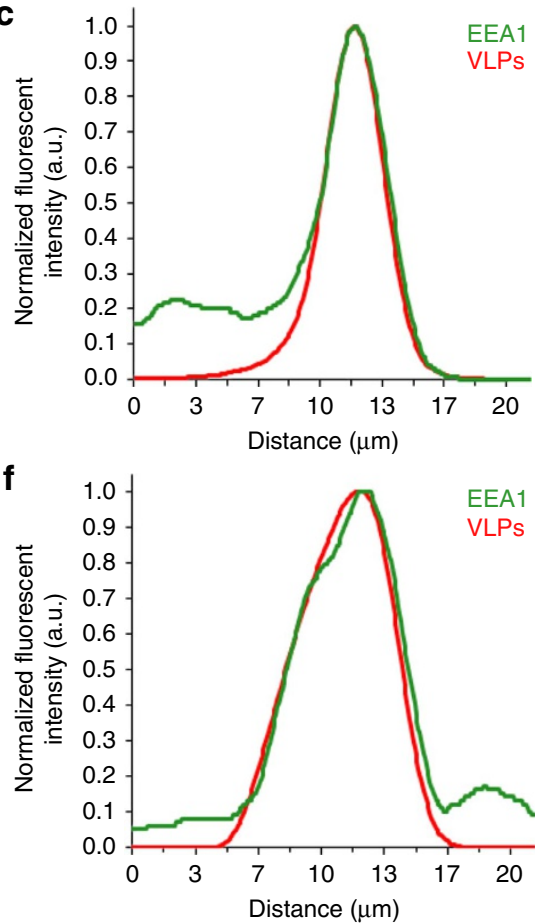

Figure 4 | Association of VP2-VLPs with EEA1-positive endosomes. Maximum intensity projections of serial confocal optical sections through Raji (a) and GM12878 cells (d) incubated overnight with VP2-VLP-AF647 (red) and treated with trypsin. White arrows represent enhanced cytoplasmic VLP localization in EEA1-positive endosomes (green). Differential interference contrast (DIC) merged with DAPI (blue) images are shown. Areas of localization (yellow) (b,e) and normalized fluorescent intensity profiles in zoomed areas $(\mathbf{c}, \mathbf{f})$ indicate VLP internalization. Scale bars, $2 \mu \mathrm{m}$.

cells released by collagenase, thus suggestive of persistence in long-lived B cells. In contrast, the viral loads of another B-cell infecting virus, Epstein-Barr ${ }^{19}$, showed no comparable difference among preparations, thus not only precluding a methodical bias as a reason for the results but also reflecting the comparatively different life cycles of these two viruses. Indeed, EBV regularly reactivates and constantly infects de novo B cells as well as several other susceptible cell populations ${ }^{20}$.

We then sorted the $\mathrm{CD}_{19}{ }^{+}$cells of individuals with the highest B19V-DNA copy numbers based on their expression of CD27 (memory) or IgD (naive) surface markers. However, the overall low B19V-DNA quantities hampered any clear resolution on the subtype of $\mathrm{B}$ cells harbouring the viral DNA. Even though not representative of the cohort, substantial data were derived solely from two individuals, ages 6 and 8, with the highest B19V-DNA copy numbers, in whom the viral DNA was found predominantly in a cluster of B cells expressing high levels of both markers. It has been suggested that in young children, germinal center-derived memory cells are preferentially of $\operatorname{IgM}$ rather than of $\operatorname{IgG}$ type $\mathrm{e}^{21}$. Thus, this B-cell subpopulation may represent $\operatorname{IgM}^{+} \mathrm{CD} 27^{+} \mathrm{IgD}^{+}$cells which have been demonstrated to be of memory type and to derive from common germinal center reactions as clonally related, class switched, $\mathrm{IgG}^{+}$memory $\mathrm{B}$ cells $^{22}$. Of note, the highest $\mathrm{B} 19 \mathrm{~V}-\mathrm{DNA}$ copies in these two individuals were found exceptionally in the monocyte fraction; yet they were B19V-IgM negative and non-viremic, suggesting non-acute albeit relatively recent primary infection. 


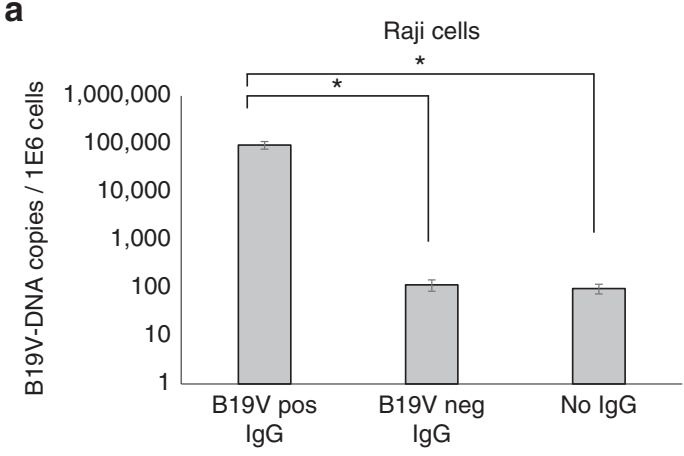

C

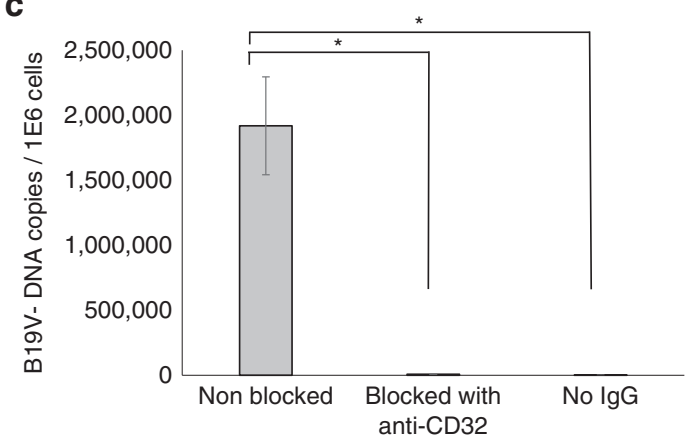

b

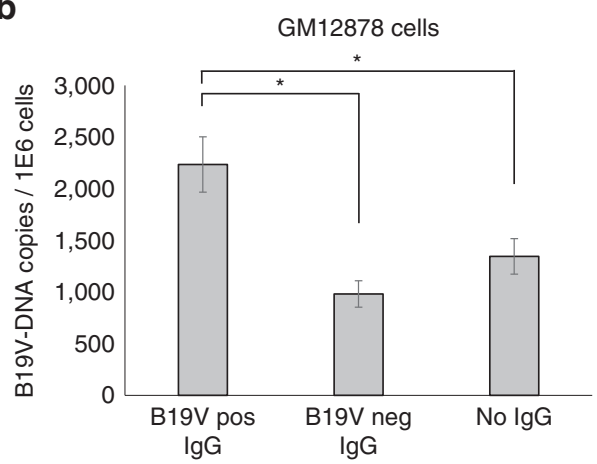

d

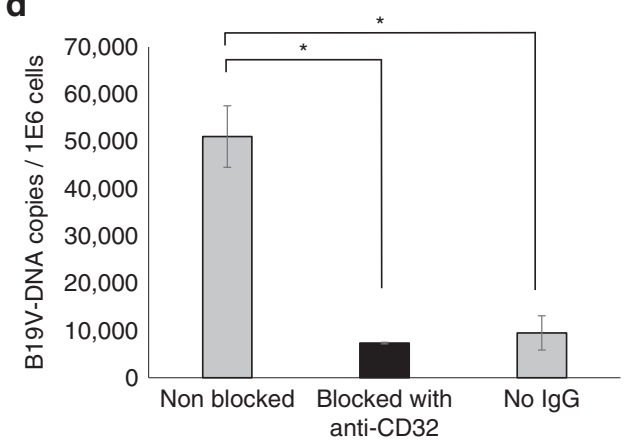

Figure 5 | Antibody-dependent uptake of native B19V in B cell lines. ADE was tested using viremic plasma in Raji (left column) and GM12878 (right column) cells in the presence of total purified B19V-positive or -negative lgGs $(\mathbf{a}, \mathbf{b})$. The effect on viral uptake was evaluated before (grey bars) and after (black bars) blocking with an anti-CD32 antibody $(\mathbf{c}, \mathbf{d})$. Error bars represent s.d. (of three replicates). ${ }^{*}$ Represents statistical significance $(P<0.001)$ between positive and respective negative controls, as calculated by Student's $t$-test.

We isolated $\mathrm{CD} 19^{+}$cells from venous blood of B19-seropositive individuals, but found the blood-derived cells to be B19V-DNA negative. This may reflect the rapid turnover of circulating $B$ cells (from a few days to 5-6 weeks) ${ }^{23}$ and/or their difference in phenotype as compared to tonsillar B cells ${ }^{24,25}$.

Even though the definitive subset of the B19V-DNA-laden $B$ cells remains to be determined, the specific genotype of B19V found provided compelling evidence of its persistence in long-lived cells. Indeed, out of the three described viral genotypes, the type 2 disappeared from circulation around 1970 (refs 3,4). In our cohort, four individuals older than 45 years carried this extinct virus type within their $B$ cells, thus alluding to the longevity of these cells. Indeed, since the B19V-DNA copy numbers in tissue wane with time and there are no detectable traces of the virus in blood or secretions other than during recent infection, it is unlikely that continuous viral replication or re-infection could explain the finding in B cells of a pathogen that circulates no more ${ }^{11}$.

Although there are some isolated case reports of B19V-type 2 viremia, particularly in immunocompromised individuals ${ }^{26,27}$, the extreme rarity of such events rules against regular replication or re-activation of the persistent virus. Also, by molecular data $\mathrm{B} 19 \mathrm{~V}$ replication has been reported to be slow or absent during persistence ${ }^{28}$ and no evidence exists of $\mathrm{B} 19 \mathrm{~V}$ integration into the host genome.

Thus, the common occurrence of the extinct type $2 \mathrm{~B} 19 \mathrm{~V}$ in our ageing individuals suggests for these very B cells a survival time of at least 40 to 60 years. This indicates that immunological memory is not only maintained in the absence of antigen re-exposure ${ }^{29,30}$ but that it is due to long-term survival of original clones.

While the presence of $\mathrm{B} 19 \mathrm{~V}$ capsid proteins has been reported in lymphoid cells of inflamed synovial tissue sections ${ }^{31,32}$, the mechanism of virus uptake into B cells has never been examined. We demonstrated the entry to be antibody mediated and CD32 dependent using both synthetic VLPs and native viruses, in line with a previous finding in monocytes ${ }^{15}$.

The CD32 receptor plays an important role in the downregulation of $\mathrm{B}$-cell responses and its deficiency or reduced expression correlates to prolonged humoral or autoimmune reactivity ${ }^{33,34}$. Analogously, B19V-related arthropathies are allegedly due to $\operatorname{IgG}$ deposition ${ }^{1}$ and this virus has been proposed to play a role in triggering or exacerbating autoimmune diseases ${ }^{35}$. Thus, our finding may provide a basis for the prolonged B-cell activation and the sustained polyclonal antibody production so characteristic of $\mathrm{B} 19 \mathrm{~V}$ acute infection ${ }^{35}$.

Moreover, B19V seropositivity has been correlated to specific patterns of gene promoter DNA methylation in children's B-cell acute lymphoblastic leukaemia ${ }^{36}$. Even without evidence of causality, it might be relevant to expand on the mechanisms by which B19V could modulate the affected B cells and whether it might play a role in the development of proliferative disorders.

In the present study, we (i) demonstrated a cellular site for $\mathrm{B} 19 \mathrm{~V}$ lifelong tissue persistence, (ii) provided a marker for the longevity of $\mathrm{B}$ cells based on the detection of a no longer circulating virus type and (iii) documented antibody-dependent enhancement as the uptake mechanism.

\section{Methods}

Tonsil collection and preparation. The right palatine tonsil and a serum sample were collected from 77 patients each, who underwent tonsillectomy/tonsillotomy for chronic tonsillitis or tonsillar hypertrophy during April-May 2015 at the Department of Otorhinolaryngology-Head and Neck Surgery of Helsinki

University Hospital. The patients' ages ranged from 2 to 69 years (mean 22). The Ethics Committee of Surgery of Helsinki and Uusimaa Hospital District 
approved the study protocol. The patients provided written consent before entry into the study.

The tonsils were collected immediately after surgery into tubes containing PBS and were kept on ice through the preparations. They were cut into smaller pieces with disposable scalpels and two separate tonsillar cell suspensions were prepared by a two-step process consisting of: (i) mechanical homogenization with a syringe plunge; (ii) collagenase digestion of the residual tonsillar tissue (from the previous step) with $0.1 \mathrm{mg} \mathrm{ml}^{-1}$ Liberase TL (Roche) at $+37^{\circ} \mathrm{C}$ for $30 \mathrm{~min}$. Each cell suspension was washed with $50 \mathrm{ml}$ PBS and filtrated through a $70 \mu \mathrm{m}$ nylon mesh (Corning Life Sciences). Henceforth, each of the cell suspensions were treated and analysed independently.

The $\mathrm{B}, \mathrm{T}$ and monocyte/macrophage cell fractions were enriched using anti-CD19, -CD3, -CD14 magnetic beads, respectively, following manufacturer's protocol (Invitrogen). The purity of the cell fractions was tested from six individuals by incubation with a 1:100 dilution of anti-human CD19-FITC (\#MHCD1910, Invitrogen), CD3-PE/cy5.5 (\#ab124052, Abcam) and CD14-PE (\#345785, BD Biosciences) on ice for $30 \mathrm{~min}$ and analysed on a BD Accuri C6 Flow cytometer.

Anti-mouse Ig, k/Negative control compensation particles set (\#552843, BD Biosciences) as well as corresponding fluorescent-labelled isotypes served as controls.

Lymphoid cell isolation from venous blood. Venous blood samples were collected from one B19V-seronegative and seven seropositive staff members. Informed consent was obtained form all study subjects and the study protocol was approved by the Ethics Committee of the Helsinki and Uusimaa Hospital District. The blood was collected using $\mathrm{BD}$ vacutainer $\mathrm{CPT}$ tubes (BD Biosciences) and the PBMCs separated as instructed by the manufacturer. From this, the $\mathrm{CD}_{1} 9^{+}$cells underwent magnetic cell separation as specified in the previous section.

DNA extraction and quantification. The DNA from tonsillar cells was extracted with the KingFisher Duo purification system (12-sample format) using the KingFisher Cell and Tissue DNA Kit (both Thermo Scientific) according to manufacturer's instructions. PBS $(n=2)$ was used as a negative control during the extractions.

B19V DNA was quantified with (i) Pan-B19V qPCR amplifying a 154 bp region of the NS1 gene and confirmed with (ii) VP-qPCR targeting a 121-bp region of the VP1 gene, as previously described ${ }^{11,37}$. The Pan-B19V qPCR products of the 33 positive individuals were purified with Diffinity RapidTip2 (Sigma-Aldrich) and Sanger sequenced with corresponding GPCR forward and reverse primers at the sequencing unit of the Institute for Molecular Medicine Finland. The sequences were analysed with Bioedit v.7.2.5 (Ibis Biosciences) and compared to B19V reference sequences (GenBank accession numbers FN598217.1 and AY044266.2 for genotype 1 and 2, respectively) using BLAST.

EBV DNA was quantified with a qPCR modified from Aalto et al. ${ }^{38}$, targeting the BALF5 DNA-polymerase gene. The EBV-qPCR reaction mix was composed of $12.5 \mu \mathrm{l}$ of $2 \times$ Maxima Probe Master Mix (Thermo Scientific) with $0.03 \mu \mathrm{M}$ of ROX passive reference dye, $0.25 \mu \mathrm{M}$ of both forward and reverse primers, $0.17 \mu \mathrm{M}$ of probe, $5 \mu \mathrm{l}$ of template DNA and nuclease-free water in a final volume of $25 \mu \mathrm{l}$. The PCR program consisted of initial denaturation at $95^{\circ} \mathrm{C}$ for $10 \mathrm{~min}$, following 45 cycles of $95^{\circ} \mathrm{C}$ for 15 seconds and $60^{\circ} \mathrm{C}$ for $1 \mathrm{~min}$. The analytical sensitivity was 10 copies/reaction

To normalize the viral DNA copies to the cell number, the human single-copy gene RNase $P$ was quantified as described ${ }^{37}$.

For quantification and as a positive control, tenfold serial dilutions (1E6-1E1 copies per $\mu \mathrm{l}$ ) of the following plasmids were used: (a) a near-full-length B19V genotype 1 genome without hairpin structures (cloned in-house) ${ }^{39}$; (b) RNase P-qPCR amplicon obtained from Dr. Janet Butel ${ }^{40}$; and (c) EBV-qPCR amplicon synthesized and cloned into the pIDTBlue vector by Integrated DNA Technologies (5'-CCCTGTTTATCCGATGGAATGACGGCGCATTTCTCGT GCGTGTACACCGTCTCGAGTATGTCGTAGACATGGAAGTCCAGAGGG CTTCCG-3').

Each sample was analysed in duplicate.

All primers and probes used in this study were ordered from Sigma Aldrich. Detailed sequence information to be found in Supplementary Table 3.

Serological studies. The serostatus of the study subjects was determined with in-house B19V-IgG and -IgM EIAs as described ${ }^{41,42}$. In brief, for the B19V-IgG EIA, $45 \mathrm{ng}$ per well of biotinylated B19-VP2-VLPs were coated onto streptavidin coated plates using sample Diluent (\#6111040, Anilabsystems, Finland). The serum samples were diluted 1:200, tested in duplicate and detected with anti-human IgG horseradish-peroxidase conjugated (Dako).

B19V-IgM antibodies were measured using an in-house IgM capture EIA as described by Maple et al. ${ }^{2}$ Microtiter plate wells (Costar, Corning) were coated with goat anti-human IgM (\#55097, Cappel/MP Biomedicals) and blocked with $3 \%$ bovine serum albumin. The serum samples were diluted 1:200 in PBS-Tween (PBST), tested in duplicate, followed by addition of $10 \mathrm{ng}$ per well of biotinylated B19-VP2-VLP. For detection, a 1:12,000 dilution of streptavidin horseradishperoxidase conjugate (Dako) was added.
Both EIAs were developed by addition of o-phenylenediamine dihydrochloride (Dako) plus $\mathrm{H}_{2} \mathrm{O}_{2}$ and the absorbances read at $492 \mathrm{~nm}$ using a Labsystems Multiscan EX (Thermo Fisher).

Cell sorting of primary tonsillar B cells. Collagenase-treated tonsillar cell suspensions were stored at $-196{ }^{\circ} \mathrm{C}$ after homogenization. Samples from 12 individuals were washed twice with PBS after thawing to remove excess DMSO, resuspended in $10 \%$ FBS-PBS solution for $10 \mathrm{~min}$ at $4{ }^{\circ} \mathrm{C}$ and stained with 1:100 dilution of anti -CD19-PE (\#ab1168, Abcam), -CD27-FITC (\#ab30366, Abcam), -IgD-APC (\#561303, BD Pharmingen) on ice in the dark for $30 \mathrm{~min}$, washed three times with PBS and immediately sorted in a BD Influx flow cytometer cell sorter.

As controls and for compensation, unstained cells, single antibodies and corresponding fluorescent-isotype controls plus anti-mouse Ig, k/Negative control compensation particles (BD Biosciences) were used.

The sorting was based on gated $\mathrm{CD} 19^{+}$cells and four cell subpopulations were collected in PBS according to the expression of either CD27 or IgD markers as: $\mathrm{CD}_{2}{ }^{+} \mathrm{IgD}^{-}, \mathrm{CD} 27^{+} \operatorname{IgD}^{\text {low }}, \mathrm{CD} 27^{+} \operatorname{IgD}^{\text {high }}$ and $\mathrm{CD} 27^{-} \operatorname{IgD}{ }^{+}$. The B19V-DNA copies were quantified from each of these subpopulations as specified above.

Cell lines. Two human B-cell lines (GM12878 and Raji (GM04671) cells, both from Coriell Institute) and a monocytic cell line, U937 (ATCC CRL-1593.2), were cultured in RPMI 1640 + GlutaMAX-I medium (Gibco), 1\% penicillinstreptomycin (Sigma-Aldrich) and complement-inactivated 10\% FBS (Gibco) at $+37^{\circ} \mathrm{C}$ and $5 \% \mathrm{CO}_{2}$.

Primary tonsillar B cells from two seronegative individuals of the study cohort were isolated with anti-CD19 magnetic beads from frozen total cell suspensions of mechanically homogenized preparations and released with DETACHaBEAD as instructed by manufacturer (Invitrogen).

Serum pools and purified human immunoglobulins. Two negative pools and a positive pool of sera $(n=5$, each) were tested before and after complement inactivation.

Total immunoglobulin $\mathrm{G}$ was purified from a B19V-IgG negative pool $(n=4)$, using the HiTrap Protein A HP column (GE Healthcare) according to manufacturer's instructions. The final IgG concentration was measured with the Pierce BCA protein assay kit (Thermo Fisher Scientific) following manufacturer's protocol.

A total purified IVIG preparation (Gammagard S/D, Baxter), of known B19V reactivity as per in-house EIA, served as positive control.

Both purified IgG preparations were complement inactivated.

Antibody-dependent enhancement. The effect of B19V specific IgGs in the internalization of VP2 VLP was tested on GM12878, Raji and U937 cell lines as well as on primary tonsillar B cells from two B19V-seronegative individuals

B19-VLPs consisting of the VP2 capsid protein were produced with the BaculoGold Baculovirus Expression System (BD Biosciences) and conjugated to Alexa Fluor 647 using a protein labelling kit (Thermo Fisher Scientific) according to manufacturer's protocol. The immunoreactivity and purity of the VP2-VLPAF647 were assessed by in-house EIA and SDS-PAGE followed by fluorescence imaging in Odyssey (LI-COR), respectively.

In each well, $1.50 \mathrm{E} 5$ of cells were cultured with $100 \mathrm{ng}$ of VP2-VLP-AF647 and incubated with either purified human IgGs or pooled sera. The purified human IgGs were used at final concentrations of $1,0.5,0.1,0.05$ or $0.01 \mathrm{mg} \mathrm{ml}^{-1}$ while the serum pools, with or without heat inactivation, were tested at dilutions of $1: 10$, $1: 50,1: 100,1: 500$ or 1:1,000. The uptake was evaluated at different time points up to $16 \mathrm{~h}$, which was then used as the standard time point for this ADE experiments. All conditions were tested in four replicates in the same plate and in at least two separate occasions. Cells only and cells combined with VP2-VLP-AF647 (without antibodies) were used as controls through the studies.

$\mathrm{ADE}$ was also tested in the $\mathrm{B}$-cell lines using live $\mathrm{B} 19 \mathrm{~V}$ from acute infection plasma. To this end, $5 \mathrm{E} 6$ cells were incubated with $10 \mathrm{~B} 19 \mathrm{~V}$ particles per cell for $40 \mathrm{~h}$ together with $1 \mathrm{mg} \mathrm{ml}^{-1}$ of either B19V-positive or -negative total purified human IgGs. All tests were carried out in triplicate and on two or more separate occasions.

Following overnight incubation, the cells were washed with cold PBS, incubated with $0.25 \%$ trypsin-EDTA (Gibco) for $15 \mathrm{~min}$ at $+37^{\circ} \mathrm{C}$, washed three times with cold $20 \%$ FBS-PBS and finally resuspended into PBS.

The efficiency of trypsin digestion was assessed by flow cytometry via expression of CD19-FITC (\#MHCD1901, Invitrogen) and CD71-FITC (\#555536, $\mathrm{BD}$ Pharmingen) as well as of cell viability with propidium iodide (Invitrogen).

After trypsin digestion, ADE was determined in the VP2-VLP-AF647 treated cultures by measuring the mean AF647 fluorescence signal with BD Accuri C6 flow cytometer (BD Biosciences).

With live B19V, DNA from the cell cultures was extracted with the DNA Blood Mini Kit (Qiagen) according to the manufacturer's instructions for viral DNA, and the B19V and RNase $P$ copies were quantified by the respective qPCRs (Pan-B19V and RNase P), as specified in DNA extraction and quantification. 
Blocking of Fc-receptor. A total of 3E6 Raji or GM12878 cells were pre-incubated with $20 \mathrm{\mu g} \mathrm{ml}^{-1}$ of anti-CD32 (\#551900, BD Biosciences) for $1 \mathrm{~h}$ at $+37^{\circ} \mathrm{C}$, then distributed to 1E5 cells per well and incubated with $100 \mathrm{ng}$ of VP2-VLP-AF647 overnight. Cells alone and cells with VLPs only were used as controls.

In the assays with live B19V, 2E6 Raji or GM12878 cells were pre-incubated with $40 \mu \mathrm{g} \mathrm{ml}^{-1}$ of anti-CD32 (BD Biosciences) for $1 \mathrm{~h}$ at $+37^{\circ} \mathrm{C}$, and then incubated with the live virus at $10 \mathrm{~B} 19 \mathrm{~V}$ particles per cell and $5 \mathrm{E} 5$ cells per well, in the presence of $1 \mathrm{mg} \mathrm{ml}^{-1}$ of purified IgGs. After $40 \mathrm{~h}$, the cells were washed and trypsinized as described above.

Confocal microscopy. VLP internalization and co-localization with intracellular structures was assessed by confocal microscopy.

After measurement of AF647 by flow cytometry, the cells were spread and air-dried on Zeiss high-performance cover slips and fixed with $4 \%$ paraformaldehyde (PFA; $20 \mathrm{~min}$ at room temperature). Early endosomal vesicles were detected with a 1:150 dilution of EEA1 mouse monoclonal antibody (\#610457, BD Biosciences), and the DNA stained during embedding with DAPI (4'-6-diamidino-2-phenylindole; Thermo Fisher Scientific), containing ProLong antifade compound. Imaging was done using an Olympus FV-1000 inverted confocal microscope (Olympus) with the UPLSAPO $\times 60$ oil-immersion objective (numerical aperture $=1.35$ ). Alexa 647 was excited with a $633 \mathrm{~nm}$ He-Ne laser and the fluorescence collected with a BA650IF long-pass filter. Alexa 488-conjugated anti-rabbit IgG was excited with a $488 \mathrm{~nm}$ argon laser and its emission detected with a 500 to $555 \mathrm{~nm}$ band-pass filter. DAPI was excited with a $405 \mathrm{~nm}$ diode laser and detected with a 425 to $475 \mathrm{~nm}$ band-pass filter. For 3D image stacks, $320 \times 320$ pixels were collected from an appropriate depth depending on the sample. Pixel resolution was adjusted to $\sim 70 \mathrm{~nm}$ per pixel in $x$ and $y$ dimensions, and to $150 \mathrm{~nm}$ in the $z$ dimension. Iterative deconvolution was performed with a signal-to-noise ratio set at 5 and a quality threshold set at 0.05 , using Huygens Essential software (SVI). Image analysis was done with Image ${ }^{43}$.

Immunolabelling of cell surface markers. The expression of $\mathrm{Fc}$ and globoside receptors was assessed on cultured Raji and GM12878 cells, as well as on CD19 cells isolated from tonsillar tissue and venous blood. Briefly, 1E6 cells were incubated with $5 \mu \mathrm{g} \mathrm{ml}^{-1}$ (1:100 dilution) of either mouse anti-human-CD32 (\#551900, BD Pharmingen) or -CD64 antibodies (\#55525, BD Pharmingen) on ice for $30 \mathrm{~min}$, washed three times with PBS and incubated with $5 \mu \mathrm{g} \mathrm{ml}^{-1}$ (1:100 dilution) of goat anti-mouse IgM/IgG-FITC secondary antibody (\#555988, BD Biosciences) for 30 mins on ice and washed three times before analysis on BD C6 Accuri.

The staining of globoside was done in a similar fashion using a 1:50 dilution of rabbit polyclonal anti-globoside GL4 (\#1960, Matreya LLC) and $5 \mu \mathrm{g} \mathrm{ml}^{-1}$ (1:100 dilution) of goat anti-rabbit-FITC conjugated secondary antibody (\#554020, BD Biosciences).

The antibody concentrations were optimized before analysis and both unstained cells as well as cells stained with secondary antibody only were used as background controls.

Statistical analysis. The median CI was calculated with normal approximation using OpenEpi v3 open source calculator. SPSS v22.0 was used to calculate the Mann-Whitney $U$ asymptotic sig. (two-sided test) and Pearson's $\chi^{2}$-test. The difference in mean fluorescence intensity in the ADE experiments was calculated with Student's $t$-test in RStudio (v3.2.4).

Data availability. All the relevant data are available from the corresponding author upon request.

\section{References}

1. Heegaard, E. D. \& Brown, K. E. Human parvovirus B19. Clin. Microbiol. Rev. 15, 485-505 (2002).

2. Qiu, J., Söderlund-Venermo, M. \& Young, N. S. Human parvoviruses. Clin. Microbiol. Rev. 30, 43-113 (2017).

3. Norja, P. et al. Bioportfolio: lifelong persistence of variant and prototypic erythrovirus DNA genomes in human tissue. Proc. Natl Acad. Sci. USA 103, 7450-7453 (2006)

4. Manning, A., Willey, S. J., Bell, J. E. \& Simmonds, P. Comparison of tissue distribution, persistence, and molecular epidemiology of parvovirus B19 and novel human parvoviruses PARV4 and human bocavirus. J. Infect. Dis. 195, 1345-1352 (2007).

5. Servant, A. et al. Genetic diversity within human erythroviruses: identification of three genotypes. J. Virol. 76, 9124-9134 (2002).

6. Sanabani, S., Neto, W. K., Pereira, J. \& Sabino, E. C. Sequence variability of human erythroviruses present in bone marrow of Brazilian patients with various parvovirus B19-related hematological symptoms. J. Clin. Microbiol. 44, 604-606 (2006).
7. Parsyan, A., Szmaragd, C., Allain, J. \& Candotti, D. Identification and genetic diversity of two human parvovirus B19 genotype 3 subtypes. J. Gen. Virol. 88, 428-431 (2007).

8. Jain, P. et al. Prevalence and genotypic characterization of human parvovirus B19 in children with hemato-oncological disorders in North India. J. Med. Virol. 87, 303-309 (2015).

9. Söderlund, M. et al. Persistence of parvovirus B19 DNA in synovial membranes of young patients with and without chronic arthropathy. Lancet 349, 1063-1065 (1997).

10. Adamson-Small, L. A., Ignatovich, I. V., Laemmerhirt, M. G. \& Hobbs, J. A. Persistent parvovirus B19 infection in non-erythroid tissues: possible role in the inflammatory and disease process. Virus Res. 190, 8-16 (2014).

11. Toppinen, M. et al. Bones hold the key to DNA virus history and epidemiology. Sci. Rep. 5, 17226 (2015).

12. Brown, K. E., Anderson, S. M. \& Young, N. S. Erythrocyte P antigen: cellular receptor for B19 parvovirus. Science 262, 114-117 (1993).

13. Weigel-Kelley, K. A., Yoder, M. C. \& Srivastava, A. $\alpha 5 \beta 1$ integrin as a cellular coreceptor for human parvovirus B19: requirement of functional activation of $\beta 1$ integrin for viral entry. Blood 102, 3927-3933 (2003).

14. Munakata, Y. et al. Ku80 autoantigen as a cellular coreceptor for human parvovirus B19 infection. Blood 106, 3449-3456 (2005).

15. Munakata, Y. et al. Human parvovirus B19 infection of monocytic cell line U937 and antibody-dependent enhancement. Virology 345, 251-257 (2006).

16. von Kietzell, K. et al. Antibody-mediated enhancement of parvovirus B19 uptake into endothelial cells mediated by a receptor for complement factor C1q. J. Virol. 88, 8102-8115 (2014).

17. Amanna, I. J., Carlson, N. E. \& Slifka, M. K. Duration of humoral immunity to common viral and vaccine antigens. N. Engl. J. Med. 357, 1903-1915 (2007).

18. Medina, F. et al. Higher maturity and connective tissue association distinguish resident from recently generated human tonsil plasma cells. J. Leukoc. Biol. 82, 1430-1436 (2007).

19. Hudnall, S. D. et al. Distribution and phenotype of Epstein-Barr virusinfected cells in human pharyngeal tonsils. Mod. Pathol. 18, 519-527 (2005).

20. Hawkins, J. B., Delgado-Eckert, E., Thorley-Lawson, D. A. \& Shapiro, M. The cycle of EBV infection explains persistence, the sizes of the infected cell populations and which come under CTL regulation. PLoS Pathog. 9, e1003685 (2013).

21. Seifert, M. \& Kuppers, R. Human memory B cells. Leukemia 30, 2283-2292 (2016).

22. Budeus, B. et al. Complexity of the human memory B-cell compartment is determined by the versatility of clonal diversification in germinal centers. Proc. Natl Acad. Sci. USA 112, E5281-E5289 (2015).

23. Fulcher, D. A. \& Basten, A. B cell life span: a review. Immunol. Cell Biol. 75, 446-455 (1997).

24. Medina, F., Segundo, C., Campos-Caro, A., Gonzalez-Garcia, I. \& Brieva, J. A. The heterogeneity shown by human plasma cells from tonsil, blood, and bone marrow reveals graded stages of increasing maturity, but local profiles of adhesion molecule expression. Blood 99, 2154-2161 (2002).

25. Perez, M. E., Billordo, L. A., Baz, P., Fainboim, L. \& Arana, E. Human memory B cells isolated from blood and tonsils are functionally distinctive. Immunol. Cell Biol. 92, 882-887 (2014).

26. Liefeldt, L. et al. Recurrent high level parvovirus B19/genotype 2 viremia in a renal transplant recipient analyzed by real-time PCR for simultaneous detection of genotypes 1 to 3. J. Med. Virol. 75, 161-169 (2005).

27. Eis-Hübinger, A. M., Reber, U., Edelmann, A., Kalus, U. \& Hofmann, J. Parvovirus B19 genotype 2 in blood donations. Transfusion 54, 1682-1684 (2014).

28. Norja, P., Eis-Hübinger, A. M., Söderlund-Venermo, M., Hedman, K. \& Simmonds, P. Rapid sequence change and geographical spread of human parvovirus B19: comparison of B19 virus evolution in acute and persistent infections. J. Virol. 82, 6427-6433 (2008).

29. Crotty, S. et al. Cutting edge: long-term B cell memory in humans after smallpox vaccination. J. Immunol. 171, 4969-4973 (2003).

30. Yu, X. et al. Neutralizing antibodies derived from the B cells of 1918 influenza pandemic survivors. Nature 455, 532-536 (2008).

31. Mehraein, Y. et al. Detection of parvovirus B19 capsid proteins in lymphocytic cells in synovial tissue of autoimmune chronic arthritis. Mod. Pathol. 16, 811-817 (2003).

32. Lennerz, C. et al. Parvovirus B19-related chronic monoarthritis: immunohistochemical detection of virus-positive lymphocytes within the synovial tissue compartment: two reported cases. Clin. Rheumatol. 23, 59-62 (2004).

33. Brownlie, R. J. et al. Distinct cell-specific control of autoimmunity and infection by Fc $\gamma$ RIIb. J. Exp. Med. 205, 883-895 (2008). 
34. Karnell, J. L. et al. CD19 and CD32b differentially regulate human B cell responsiveness. J. Immunol. 192, 1480-1490 (2014).

35. Kerr, J. R. The role of parvovirus B19 in the pathogenesis of autoimmunity and autoimmune disease. J. Clin. Pathol. 69, 279-291 (2016).

36. Vasconcelos, G. M. et al. History of parvovirus B19 infection is associated with a DNA methylation signature in childhood acute lymphoblastic leukemia. Epigenetics 6, 1436-1443 (2011).

37. Toppinen, M. et al. A new quantitative PCR for human parvovirus B19 genotypes. J. Virol. Methods 218, 40-45 (2015).

38. Aalto, S. M. et al. Lymphoproliferative disease after allogeneic stem cell transplantation--pre-emptive diagnosis by quantification of Epstein-Barr virus DNA in serum. J. Clin. Virol. 28, 275-283 (2003).

39. Brunstein, J., Söderlund-Venermo, M. \& Hedman, K. Identification of a novel RNA splicing pattern as a basis of restricted cell tropism of erythrovirus B19. Virology 274, 284-291 (2000).

40. McNees, A. L., White, Z. S., Zanwar, P., Vilchez, R. A. \& Butel, J. S. Specific and quantitative detection of human polyomaviruses BKV, JCV, and SV40 by real time PCR. J. Clin. Virol. 34, 52-62 (2005).

41. Kaikkonen, L. et al. Acute-phase-specific heptapeptide epitope for diagnosis of parvovirus B19 infection. J. Clin. Microbiol. 37, 3952-3956 (1999).

42. Maple, P. A. C. et al. Identification of past and recent parvovirus B19 infection in immunocompetent individuals by quantitative PCR and enzyme immunoassays: a dual-laboratory study. J. Clin. Microbiol. 52, 947-956 (2014).

43. Abràmoff, M., Magalhães, P. \& Ram, S. Image processing with ImageJ. Biophoton. Int. 11, 36 (2004).

\section{Acknowledgements}

We thank Petriina Mannelli for help with tissue collection, Visa Nurmi and Jussi Hepojoki for providing serum samples and fluorophore-labelled VLPs, Lassi Palmujoki for help with confocal imaging, Prof. Jukka Pelkonen for scientific guidance, Pieta Mattila for comments on the manuscript, Kalle Kantola for technical support with figures and the Biomedicum FACS core facility where the flow cytometry and cell sorting were performed. This work was funded by the Academy of Finland (grant 1257964), the Jane and Aatos Erkko Foundation (K.H. and M.V.-R.), the Sigrid Jusélius Foundation, the
Helsinki University Hospital Research \& Education Fund, the Finnish-Norwegian Medical Foundation, the Medical Society of Finland (FLS), the Research Funds of the University of Helsinki and the Life and Health Medical Grant Association.

\section{Author contributions}

L.P., M.T., E.M., L.H., M.F.P. performed the experiments; L.-M.A. and T.I. provided serum and tissue samples and clinical data; L.P., M.T., E.M., M.F.P. performed the data analysis; K.H., M.S.-V., M.V.-R., M.F.P. contributed to the study design; all authors participated in interpretation, manuscript writing and editing.

\section{Additional information}

Supplementary Information accompanies this paper at http://www.nature.com/ naturecommunications

Competing interests: The authors declare no competing financial interests.

Reprints and permission information is available online at http://npg.nature.com/ reprintsandpermissions/

How to cite this article: Pyöriä, L. et al. Extinct type of human parvovirus B19 persists in tonsillar B cells. Nat. Commun. 8, 14930 doi: 10.1038/ncomms14930 (2017).

Publisher's note: Springer Nature remains neutral with regard to jurisdictional claims in published maps and institutional affiliations.

(c) (i) This work is licensed under a Creative Commons Attribution 4.0 International License. The images or other third party material in this article are included in the article's Creative Commons license, unless indicated otherwise in the credit line; if the material is not included under the Creative Commons license, users will need to obtain permission from the license holder to reproduce the material. To view a copy of this license, visit http://creativecommons.org/licenses/by/4.0/

(C) The Author(s) 2017 